\title{
Designing Anchored Instructions via Virtual Role Playing
}

\author{
Xin Bai*, Joanne Lavin \\ Teacher Education / Educational Technology, York College of the City University of New York, 94-20 Guy R. \\ Brewer Blvd., Jamaica NY 11451 USA. \\ CUNY SPS RN-BS Nursing Program, 101 West 31st Street, New York, NY 10001 USA. \\ * Corresponding author. Tel.: 718-262-2830; email: xbai@york.cuny.edu \\ Manuscript submitted July 28, 2017; accepted May 4, 2018. \\ doi: 10.17706/ijeeee.2019.9.1.1-8
}

\begin{abstract}
Authentic learning activities were anchored in a virtual hospital setting in our study. Students watched a 3D case study, role-played, and created concept maps to externalize their thoughts. Their knowledge and skills were measured via traditional multiple-choice questions, role playing, and concept mapping. Pre- and post-tests showed no significant difference between control and treatment groups. However, the analyses of the role-playing and concept maps demonstrated the treatment group gained significantly. Strengths and constraints of role playing are discussed and important issues implied are included to help understand effective situated learning instructional design strategies in an authentic learning environment.
\end{abstract}

Key words: Role playing, simulation, educational technology, assessment.

\section{Introduction}

Researchers have explored the potential of anchoring learning in a virtual community as an alternative to a real world setting to promote authentic learning, which allows students to observe how other people behave and participate themselves under various conditions [1], [2]. Such approaches are found to be an effective instructional strategy that can enhance students' transition from classroom to real-life practice, provide opportunities for students to analyze information, make clinical judgments, and respond as if working with actual patients [3]. Many articles in the nursing literature identify the benefits of using various types of simulations to enhance self-confidence as well as skill performance [4], [5]. Additionally collaborative learning in nursing promotes clinical judgment [6]. Zitzelsberger et al reported the use of wikis to promote collaborative learning [7]. The results have led to this study, which explores what kind of learning activities occur in a virtual learning setting, how students respond to such experiences, and what kind of evaluation tools are effective in measuring learning outcomes.

\section{Theoretical Framework}

\subsection{Anchored Instructions}

Anchored instruction falls under the social constructivism paradigm. It involves the use of a piece of media (e.g., a video) to create a shared experience among learners [8]. It often serves as a starting point for further learning. Social learning theorist Bandura states that people learn through observing other's behavior, attitude, and outcomes of those behaviors [9]. The observed behaviors can later serve as a guide for action. Social learning theorists try to understand how people learn effectively via continuous reciprocal 
interaction involving attention, memory, and motivation. Factors that affect the amount of attention include one's sensory capacities, arousal level, an object or event's distinctiveness, affective valence, prevalence, complexity, functional value, to name to few [10], [11]. Vygotsky's social learning development theory asserts social interaction plays a fundamental role in the process of cognitive development. A student learns when engages in shared experiences with a more knowledgeable other who has a better understanding or a higher ability level [12]-[14]. Vygotsky's situated learning also emphasizes the importance of social learning. He argues that learning should be situated within authentic activity, context, and culture. Thus knowledge needs to be presented in authentic contexts that would normally involve that knowledge [15], [16].

Learning in a real-life like environment allows students to behave and observe with peers and domain experts, the process of which results in knowledge co-construction. However, it is usually not practical or possible to situate students in a real life setting to deal with problems due to the constraints of costs, space, or risks in a traditional classroom. More research is needed to harness the advances of technologies in order to provide our students with a meaningful learning context similar to real life.

\subsection{Role Playing in a Virtual Setting}

Role-playing can meet the broad teaching goals of thinking critically, communicating effectively, and intervening therapeutically [17]. Students can assume a new role, learn by doing and reflection, take risks, and engage in meaningful knowledge construction through proactive interaction with peers in the process. Many organizations recommend the use of role-playing in an authentic setting especially for team building [18]. Debriefing after role-playing is important in the process of developing clinical judgment skills [19]. Assessing whether learning can transfer to a new problematic situation becomes critical in designing the virtual environment and develop anchored instructions.

\subsection{Learning Assessment}

Learning Assessment provides feedback to teachers and students that allows them to modify the teaching and learning activities in which they are engaged [20]. An an authentic learning setting, problems are presented as those in the real world. This is an educational ideal as the goal of education is to prepare students to be competent professionals and responsible citizens in the real world. Thus authentic assessment occurs most naturally when in context and when assessment addresses genuine concerns and problems of the students [21]. Traditional assessment tools such as multiple-choice questions focus mainly on content knowledge. They often fail to evaluate the real world problem solving skills that require students to interact with others to identify and articulate a problem, develop best solutions, and share the findings with others to optimize the results.

\section{Virtual Field Trip Design for Role Playing}

Researchers in Nursing first wrote a case study script on the topic of Strokes (CVA) and designed assessment instruments. The plan was to play out this scenario and videotape it as a 3D teaching vignette to be used in basic baccalaureate nursing curriculum as well as in acute care hospital settings for Registered Nurse (RN) continuing education. The case is about an older woman who develops a stroke and follows her emergency admission through discharge. The woman has many of the risk factors for strokes: she did not consistently take her medications nor follow the recommended diet. The story guides students through the assessment and acute management of a stroke. Constant communication among the researchers and domain experts are established to achieve a better understanding of domain specific contents such as NIHH/Glasgow Scales or t-PA. The NIH stroke scale (NIHSS) measures several aspects of brain function, including consciousness, vision, sensation, movement, speech, and language. A certain number of points are given for each impairment uncovered during a focused neurological examination. For instance, in the exam 
the patient is asked to open/close eyes, lift a leg, open/close mouth, squeeze hand, read words/sentences or describe a picture.

The researchers evaluated Second Life, OpenSim, SimCity, Flash, Director, and Unreal. They narrowed down to a MUVE, Second Life, as it provides virtual reality contexts that support social interaction such as role-playing. Second Life does not embed explicit game rules or game-based rewards like those in commercial multi-player online games. But it supports for constructivist learning by allowing for meaningful knowledge construction from a first-person perspective in a social setting [22]-[24]. Second Life is a user-friendly 3D simulation platform. Little or no prior gaming experience is needed for users to learn how to navigate and interact with others. Users can create buildings, roads, bridges as well as other artifacts such as vehicles, trees, animals, or even zombies. The basic unit of these virtual items is a primitive, or prim, which is represented by a set of parameters including shape, position, size, rotation, hollow, twist, etc. There is a built-in authoring tool that allows users to construct 3D objects made out of prims and design behaviors via scripts. Second life was therefore adopted as the researchers' platform for developing 3D teaching vignettes. A virtual clinic was built that included a front desk with a waiting area, an observation room with equipment such a heart monitor, ultrasound machine, a CT scanner, a sink to wash hands, cabinets with medical instruments, and hospital beds with curtains.

\section{Research Methods}

\subsection{Participants and Procedures}

Twenty undergraduate Nursing students at an urban public university were recruited and randomly assigned to one of the four groups with five students in each group. After signing a consent form, they took a pre-test for prior knowledge, followed by a brief orientation by a faculty member from Nursing on Strokes. Then Groups $1 \& 2$ read a paper-based case in traditional text; Groups 3\& 4 watched the same case in video format (Table 1). All the groups then completed Question Set A. Next, they were asked to role-play via text-chatting online (Group $1 \& 3$ ) or 3D simulation online (Group $2 \& 4$ ) to address another complex inter-professional problem.

Table 1. Study Design (N=20; 4 Groups with 5 Participants in Each Group)

\begin{tabular}{|c|c|c|c|c|c|}
\hline Steps & Pre-test & Orientation & $\begin{array}{l}\text { Case Study (CS) } \\
\text { (Followed by Ques. Set A) }\end{array}$ & $\begin{array}{l}\text { Role-Play (RP) } \\
\text { (Followed by Ques. Set B) }\end{array}$ & Post-test \\
\hline Duration & $(15 \mathrm{~min})$ & $(15 \mathrm{~min})$ & (30 min.) & (50 min.) & (20 min.) \\
\hline Group 1 & & & Read case via text & Role-play via text chatting online & \\
\hline Group 2 & & & Read case via text & Role-play via 3D simulations & \\
\hline Group 3 & & & Watch case via simulation video & Role-play via text chatting online & \\
\hline Group 4 & & & Watch case via simulation video & Role-play via 3D simulations & \\
\hline
\end{tabular}

Each of them was given a card describing the role they are playing (Fig. 1). Afterwards, they completed Question Set B, which included a concept mapping.

\subsection{Assessment Instruments}

The assessment instruments include: (1) pre- and post-tests, (2) Question Set A \& B, and (3) role-playing records (online chatting logs or videotaped activities). Pre- and Post-tests are the same for each group, which include multiple-choice questions and open-ended questions (measuring domain knowledge), as well as survey questions (measuring attitudes). For instance, some questions are "How should a nurse assess for evidence of facial palsy?" "tPA (Tissue Plasminogen Activator) is given in $0.9 \mathrm{mg} / \mathrm{kg}$. A patient 
weighs 142 pounds $(64.5 \mathrm{~kg}$ ). Please select the correct total dose of TPA that is to be given." Question Set A is largely factual and procedural (e.g. "Risk factors that may lead to strokes" "What exams need to be scheduled within the first 25 minutes?"). Question Set B uses inference questions (e.g. "Why there is a need to communicate with the relatives of stroke patients?" and "How should a nurse manage psychosocial needs of stroke patients?). Each question was scored on 3-point scale (1: incorrect/no answer, 2: partially correct but incomplete, 3: precise and detailed). We conducted a mixed or split plot design, in which CS and RL are crossed between-subject factors. Each assessment instruments (i.e., Pre-test, Question Set A, B, and Post-test) was a within-subject factor on the dependent measurement of learning outcomes. We looked for possible two-way interaction among CS, RP, and Assessment.

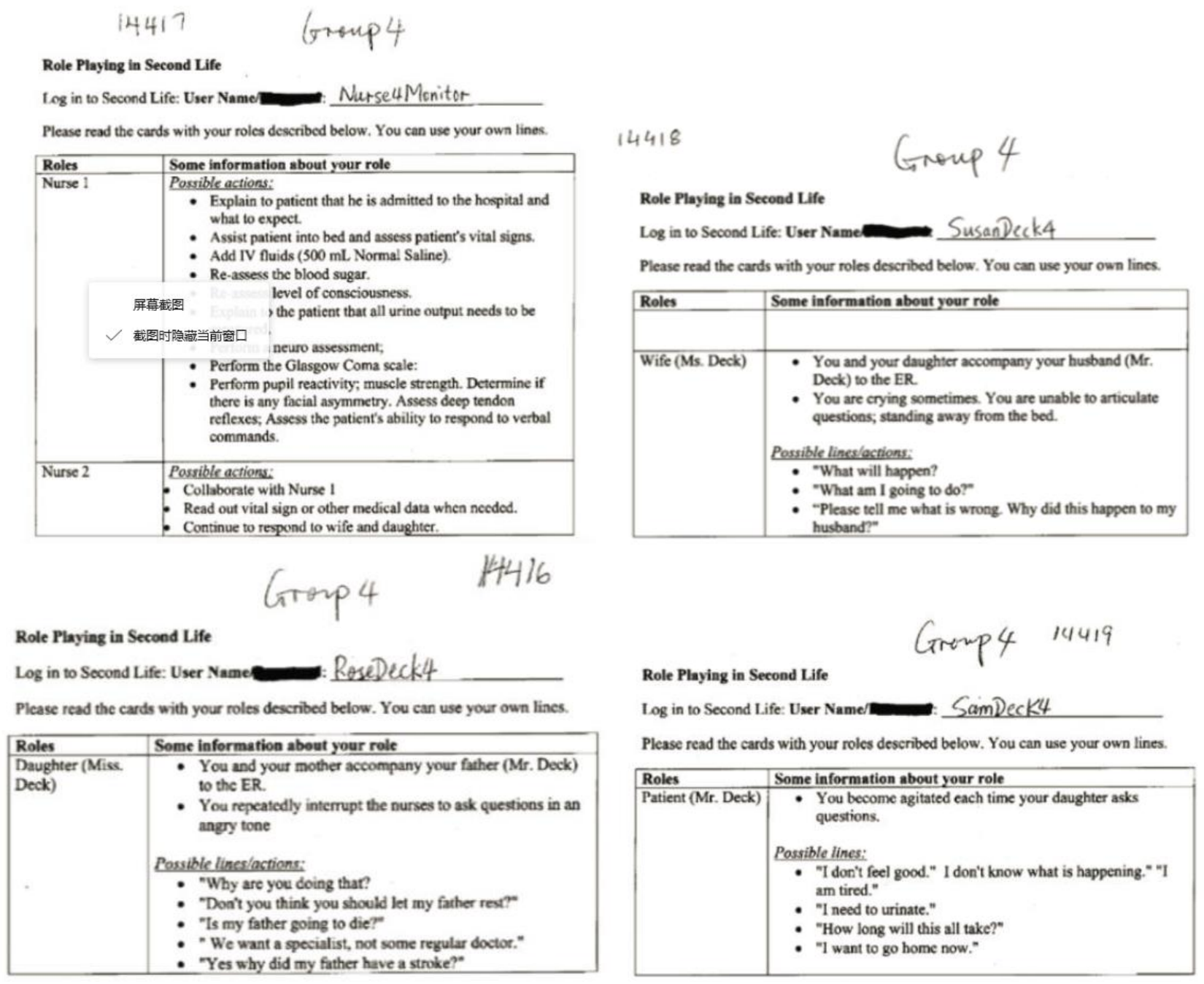

Fig. 1. Role play cards - one for each role player. It includes a patient, wife, daughter, and nurses.

To analyze the role-playing activities, raters examined the videotapes and text-chatting logs. Qualitative analysis via thematic coding and member checking were conducted.

\subsection{Role Playing Analysis Results}

We recorded the interactions during students' role playing session. We set up several cameraman avatars in the virtual setting controlled by our research assistant. Each cameraman observed and videotaped a group's interactions from his/her perspective (Fig. 2). 


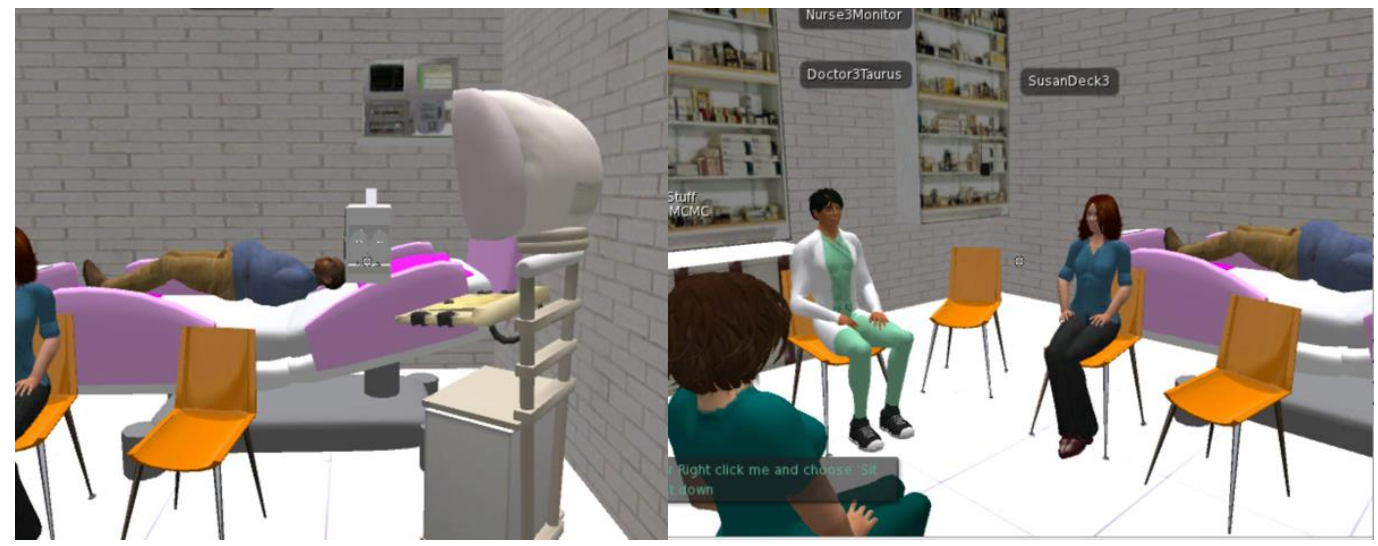

Fig. 2. Role play-visual social interactions.

The social knowledge co-construction process during role playing was analyzed via the coding scheme of the Interaction Analysis Model (IAM) [25], to understand learners' interactive social knowledge co-construction behaviors [26]-[29]. Each turn of student conversations was treated as one unit and classified into five themes within IAM. These themes are sharing, comparing of information; discovery of dissonance; negotiation of meaning/co-construction of knowledge; testing and modification of proposed synthesis; agreement or application of newly constructed meaning (Fig. 3).

The Text Group generated 195 nodes; while the Virtual Group generated 294 nodes. This indicates the Virtual Group participated much more than the Text Group during the virtual field trip, even though they had a steeper learning curve navigating in the new virtual environment, Second Life. The irrelevant information (S6: Others) is extremely low in both groups (7 vs. 7), suggesting both groups were engaged in role-playing productively throughout the field trips.

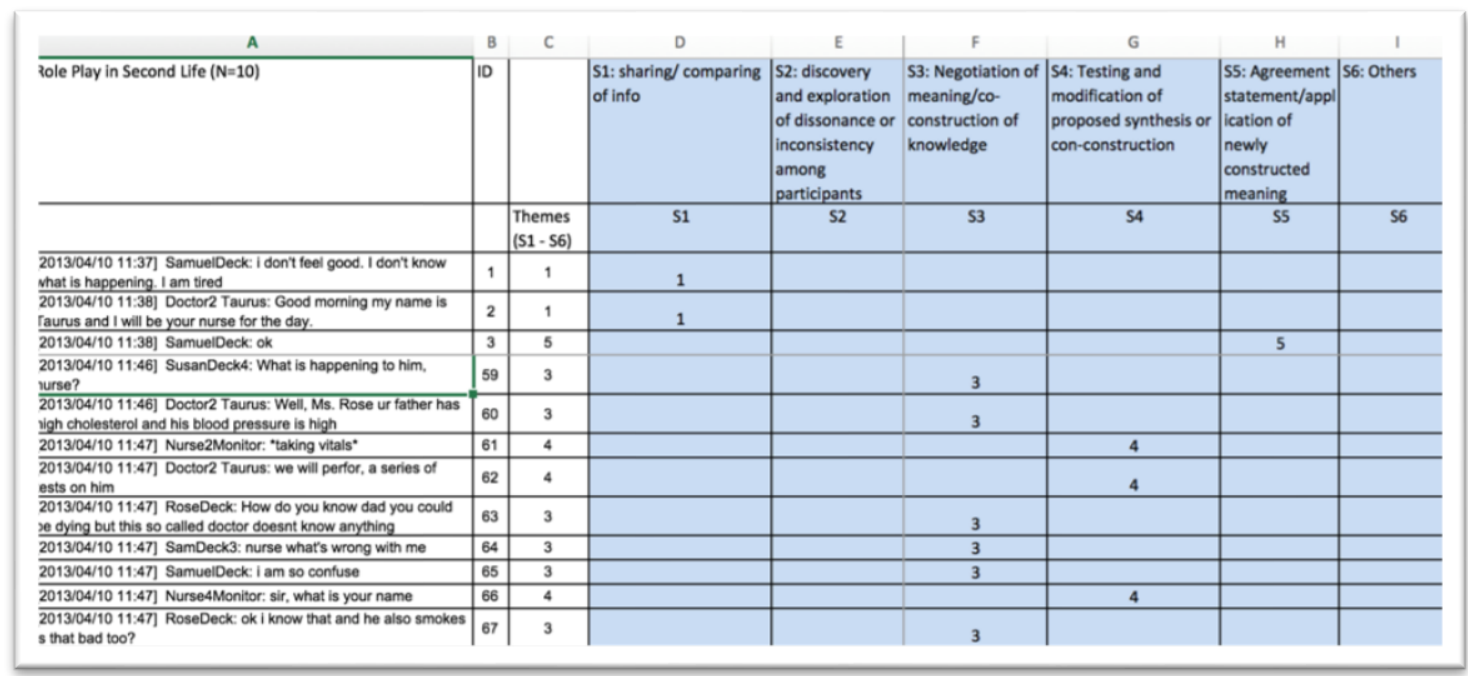

Fig. 3. Role play-verbal conversation analysis.

\section{Discussion}

Role playing creates a student-centered learning environment where a student learns through articulating the view point of a character, who may have emotional or physical needs. This interactive learning opportunity not only allows users to be actively engaged, it immerses them in an authentic context with constant self and peer assessment, thus obtaining valuable formative feedback. 
Although pre- and post-tests result shows that there is no significant main effect of the treatment among the four groups. Role Playing analysis (via conversation logs) shows Virtual Group participated much more than the Text Group during the virtual field trip. Also, the virtual group focused much less on S1 (sharing and comparing) than the text group (67 vs. 46), but more on other higher-level aspects of social interactions. This suggests that the Text Group explored less on knowledge co-construction with other stakeholders and they were not able to build-on to each other's ideas. Instead, they ask questions or clarify ideas without further negotiations. The Virtual Group consistently build knowledge in all the phases of social interactions. The concept mapping analysis result shows that the visual/visual group gained significantly the most. The correct number of responses more than doubled than those from the rest of the three groups in all the categories. This shows concept mapping, as an assessment tool, is able to measure student learning in a different perspective than the traditional multiple-choice questions. The results above lead to the following questions: Why the different assessment instruments generated different results? What kind of learning skills is being measured by each instrument? Which instrument(s) can effectively measure what we intend to evaluate?

One explanation of this result could be that the assessment questions used in pre and posttests (i.e., 20 multiple-choice questions) mostly assessed students' short-term declarative knowledge learning. It may not be the best method to determine whether students can transfer their learning to new situations, which was assessed in role-playing and concept mapping. In order for students to demonstrate how they solve problems in real life, a better way to assess students' learning could be through solving problems in the virtual environment via communicating with others - role playing. The different assessment results demonstrate that varied assessment instruments are needed to assess what and how students have acquired knowledge and skills in a virtual learning environments. Such skills include problem-solving, communication, or cultural diversity awareness, which are essential in the diverse globalized world. Additionally, as people show preference for social stimuli in the information processing circuits in the brain, we suggest that a combination of information processing and sociocultural perspectives be considered for effective learning assessment.

\section{Conclusion}

Learning based upon anchored instructions should be assessed through multiple perspectives. We identify the strengths and weaknesses of several assessment instruments and discuss their implications. Our study indicates that role-playing can serve as effective learning and assessment tools for measuring students' situated learning in a relevant and motivating virtual environment. Role-playing promotes communication, problem solving, clinical reasoning, and planning skills. Further investigate is needed to explore the implications of promoting situated learning through virtual role playing and the underlining instructional design principles in a 3D virtual world.

\section{References}

[1] Dede, C., Salzman, M., \& Loftin, R. B. (1996). The development of a virtual world for learning Newtonian mechnics. In P. Brusilovsky, P. Kommers, \& N. Streitz (Eds.), Multimedia, Hypermedia, and Virtual Reality, 87-106. Berlin: Springer

[2] Hew, K., \& Cheung, W. (2008). Use of three-dimensional (3-D) immersive virtual worlds in K-12 and higher education settings: A review of the research. British Journal of Educational Technology, 41(1), 33-55.

[3] Stokowski, L. A. (2013). Digital revolution: Games, simulations, and virtual worlds in nursing education. Medscape. WebMD, LLC 
[4] Galloway, S. J. (2009) Simulation techniques to bridge the gap between novice and competent healthcare professionals OJIN. The Online Journal of Issues in Nursing, 14(2).

[5] Wotton, K., Davis, J., Button, D., \& Kelton, M. (2010). Third year undergraduate nursing students' perceptions of high fidelity simulation. Journal of Nursing Education, 49(11), 632-639.

[6] Galt, K. (2015). Implementing collaborative learning in prelicensure nursing curricula: Student perceptions and learning outcomes. Nurse Educator, 40(4), 183-188.

[7] Zitzelsberger, H., Campbell, K., Service, D., \& Sanchez, O. (2015). Using wikis to stimulate collaborative learning in two online health sciences courses. Journal of Nursing Education, 54(6), 352-355.

[8] Bransford, J. D., Sherwood, R. D., Hasselbring, T. S., Kinzer, C. K., \& Williams, S. M. (1990). Anchored instruction: Why we need it and how technology can help. Cognition, Education, and Multimedia: Exploring Ideas in High Technology, 115-141.

[9] Bandura, A. (1977). Social Learning Theory. New York: General Learning Press.

[10] Bandura, A. (1973). Aggression: A Social Learning Analysis. Englewood Cliffs, NJ: Prentice-Hall.

[11] Bandura, A., \& Walters, R. (1963). Social Learning and Personality Development. New York: Holt, Rinehart \& Winston

[12] Vygotsky, L. S. (1980). Mind in Society: The Development of Higher Psychological Processes. Harvard university press.

[13] Bai, X., \& Lavin, J. (2016) Evaluating social learning in a virtual environment via concept maps. International Journal of Learning and Teaching, 2(2)

[14] Crawford, K. (1996). Vygotskian approaches in human development in the information era. Educational Studies in Mathematics, 31(1-2), 43-62.

[15] Lave, J. (1988). Cognition in Practice: Mind, Mathematics, and Culture in Everyday Life. Cambridge, UK: Cambridge University Press.

[16] Lave, J., \& Wenger, E. (1990). Situated Learning: Legitimate Peripheral Participation. Cambridge, UK: Cambridge University Press.

[17] Daley, K. \& Campbell, S. H. (2008). Framework for simulation learning in nursing education. Simulation Scenarios for Nurse Educators Making It Real. New York: Springer Publishing Company.

[18] Paige, J. B., \& Daley, B. J. (2009). Situated cognition: A learning framework to support and guide high-fidelity simulation. Clinical Simulation in Nursing, 5(3), e97-e103.

[19] Sweigart, L., \& Hodson-Carlton, K. (2013). Improving student interview skills: The virtual avatar as client. Nurse Educator, 28 (1), 11-15.

[20] Black, P., \& Wiliam, D. (1998). Assessment and classroom learning. Assessment in Education: Principles, Policy \& Practice, 5(1), 7-74.

[21] Brooks, J. G., \& Brooks, M. G. (1993). In search of understanding: The case for constructivist classrooms. Alexandria. VA: Association for Supervision and Curriculum Development.

[22] Bricken, M., \& Byrnes, C. M. (1993). Summer students in virtual reality: a pilot study on educational applications of virtual reality technology. In A. Wexelblat (Ed.), Virtual Reality: Applications and Explorations, (pp. 199-217). Boston, MA: Academic.

[23] Dede, C. (1995). The evolution of constructivist learning environments: Immersion in distributed virtual worlds. Educational Technology, 35(5), 46-52.

[24] Winn, W. (1997). The impact of three-dimensional immersive virtual environments on modern pedagogy. HITL Report R-97-15.

[25] Gunawardena, C., Lowe, C., \& Anderson, T. (1997). Analysis of global online debate and the development of an interaction analysis model for examining social construction of knowledge in computer conferencing. Journal of Educational Computing Research, 17(4), 397-431. 
[26] Hou, H. (2012). Analyzing the learning process of an online role-playing discussion activity. Educational Technology \& Society, 15(1), 211.

[27] Hou, H. T., Chang, K. E., \& Sung, Y. T. (2007). An analysis of peer assessment online discussions within a course that uses project-based learning. Interactive Learning Environments, 15(3), 237-251.

[28] Jeong, A. C. (2003). The sequential analysis of group interaction and critical thinking in online threaded discussions. American Journal of Distance Education, 17(1), 25-43.

[29] Sing, C. C., \& Khine, M. S. (2006). An analysis of interaction and participation patterns in online community. Educational Technology \& Society, 9(1), 250-261

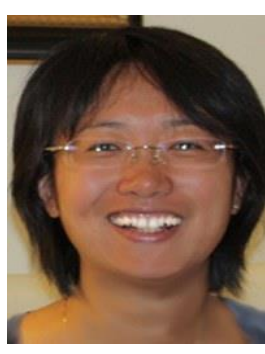

Xin Bai is an associate professor of Educational Technology in Department of Teacher Education at York College, City University of New York. She earned her doctorate in Instructional Technology \& Media from Teachers College at Columbia University. Her research focuses on educational games, simulations, intelligent tutoring systems, and e-learning. Her work is built on the research done on cognitive science, artificial intelligence, and educational technologies.

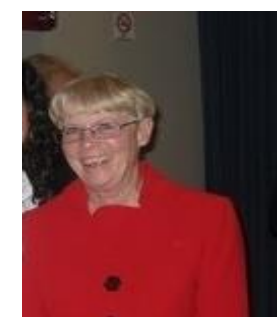

Joanne Lavin is RN, EdD, and board certified clinical specialist in psychiatric mental health nursing, serves as the associate director of nursing at CUNY SPS. She joins SPS from York College where she served as professor and director of nursing programs. Previously, she had been a professor of nursing at Kingsborough Community College, also serving as deputy chair and chair of the department since 1996. Dr. Lavin holds an EdD and MEd in nursing education from Teachers College, an MS in community health education from Hunter College, a BS in nursing from Regents College, a BS in biology from Marymount Manhattan College, and a diploma from St. Mary's Hospital School of Nursing. 